\title{
Computing the merger of black-hole binaries: The IBBH problem
}

\author{
Patrick R. Brady, Jolien D. E. Creighton, and Kip S. Thorne \\ Theoretical Astrophysics, California Institute of Technology, Pasadena, California 91125
}

(Received 22 April 1998; published 26 August 1998)

\begin{abstract}
Gravitational radiation arising from the inspiral and merger of binary black holes (BBH's) is a promising candidate for detection by kilometer-scale interferometric gravitational wave observatories. This Rapid Communication discusses a serious obstacle to searches for such radiation and to the interpretation of any observed waves: the inability of current computational techniques to evolve a BBH through its last $\sim 10$ orbits of inspiral $(\sim 100$ radians of gravitational-wave phase). A new set of numerical-relativity techniques is proposed for solving this "intermediate binary black hole" (IBBH) problem: (i) numerical evolutions performed in coordinates co-rotating with the $\mathrm{BBH}$, in which the metric coefficients evolve on the long timescale of inspiral, and (ii) techniques for mathematically freezing out gravitational degrees of freedom that are not excited by the waves. [S0556-2821(98)50218-4]

PACS number(s): 04.25.Dm, 04.30.Db, 04.70.-s
\end{abstract}

\section{MOTIVATION}

Among all gravitational wave sources that theorists have considered, the one most likely to be detected first is the final inspiral and merger of binary black holes (BBH's) with masses $M_{1} \sim M_{2} \sim 10-20 M_{\odot}[1]$. Detailed analyses of the evolution of stellar and black-hole populations [2] predict event rates as high as $\sim$ one per year in the first LIGO and VIRGO interferometers (2002-2003) and a thousand per year in enhanced interferometers for which research and development is currently under way, but the rates could also be far lower than this.

Optimal search techniques require prior information about the gravitational waveforms. The waveforms from the early binary inspiral phase, when the holes are far apart, are calculated by a post-Newtonian (PN) expansion [3]. The merger phase, beginning at the innermost stable circular orbit, will be calculated by numerical relativity. Unfortunately, there is a gap [4] between the failure of the PN expansion (which, for concreteness, we take to occur when its Taylor series makes a $2 \%$ error in the energy loss rate [5]) and the beginning of merger. Filling this gap is called the intermediate binary black hole (IBBH) problem [4].

We estimate [5] the PN failure point, for calculations at 3PN order $\left[\mathrm{O}\left(v^{6}\right)\right.$ beyond Newtonian gravity and quadrupolar radiation reaction], to be at the orbital speed $v$ $\equiv(M \Omega)^{1 / 3} \simeq 0.3$ (where $M$ is the system's total mass, $\Omega$ is its orbital angular velocity, and $G=c=1$ ); there the remaining time to merger, remaining number of orbits, and remaining number of gravitational-wave radians are $T$ $\simeq 1200 M, N_{\text {orbits }} \simeq 8$, and $\Phi \simeq 100$. For $2.5 P N$ calculations, the PN failure is at $v \simeq 0.25$ where $T \simeq 5000 M, N_{\text {orbits }} \simeq 20$, and $\Phi \simeq 250$. For optimal detection of the waves, the waveform must be accurately modeled in the IBBH gap [5]. The wave frequency in this gap is $f=\Omega / \pi \sim(50-$ $200 \mathrm{~Hz})\left(20 M_{\odot} / M\right)$, which is the band of optimal LIGO and VIRGO sensitivity. This adds urgency to the IBBH problem.

For numerical simulations of the merger phase, the conventional approach uses asymptotically inertial coordinates in which the dynamical timescale, $\tau_{\text {dyn }} \sim M$, is set by the task of moving the holes across the coordinate grid. It is unlikely that, in the next several years, this approach will be able to evolve a BBH through the gap for the required $\gtrsim 1200 \mathrm{dy}-$ namical time scales. This motivates exploring alternative procedures for computing the evolution and waves during the IBBH phase.

One possible method of extending the PN approximation to the waveform into and through the IBBH region is to augment it with Padé approximants [6]. However, there is little hope, via PN Padé approximants, to evolve the binary's internal spacetime geometry in the IBBH region and thereby provide (i) initial data for numerical relativity's analysis of the merger, and (ii) a connection between those initial data and the binary's early inspiral properties (masses, spins, orbit). For these crucial issues we must turn elsewhere.

Here we explore an alternative strategy [4]: numerical relativity computations performed not in asymptotically inertial coordinates (as is normally done), but instead using spatial coordinates which co-rotate with the holes' orbital motion and a temporal slicing which adjusts, as the potential well between the holes deepens, so as to keep all the metric coefficients as slowly evolving as possible. In such coordinates one hopes to achieve a timescale $\tau_{\text {dyn }}$ for dynamical evolution of the metric coefficients that is of the order of the timescale $\tau_{*}$ on which radiation reaction drives the holes together. ${ }^{1}$ Since the orbital frequency changes by only a factor of $\sim 2-3$ through the IBBH phase, this phase may last only $\sim 3$ dynamical timescales in the co-rotating frame-an enormous reduction from the $\gtrsim 1200$ timescales in the asymptotically inertial coordinates of standard numerical relativity.

Although the metric coefficients' true dynamical timescale will be $\tau_{*}$ in these co-rotating coordinates, numerical approximations may excite spurious gravitational waves with

\footnotetext{
${ }^{1}$ If the holes are spinning with axes inclined to the orbital angular momentum, then in these coordinates the evolution timescale may be shorter: $\tau_{*} \sim$ (spins' precession period). For simplicity we shall ignore this possibility, though our analysis presumably can be adapted to handle it.
} 
wavelengths of the order of the spatial grid size. A good numerical scheme must freeze out these unphysical modes in order to achieve stable evolution while using long time steps. Correspondingly, a concrete implementation of our strategy must include two elements: first, a method to choose the lapse and shift so the coordinates co-rotate with the binary; second, a numerical scheme that evolves stably with time steps constrained only by $\tau_{*}$. Such a scheme differs from that of previous co-rotating neutron-star-binary calculations [7], which have not evolved the gravitational field but instead computed sequences of equilibria.

\section{CHOOSING THE LAPSE AND SHIFT}

Numerical relativity is based on a $3+1$ decomposition of the metric:

$$
d s^{2}=-\alpha^{2} d t^{2}+\gamma_{i j}\left(d x^{i}+\beta^{i} d t\right)\left(d x^{j}+\beta^{j} d t\right) .
$$

Here $\alpha$ is the lapse function, $\beta^{i}$ is the shift vector, and $\gamma_{i j}$ is the metric of the 3-dimensional slices of constant time $t$. The lapse and shift are specified freely during the evolution, thereby fixing the spacetime coordinates.

We propose to construct the initial IBBH co-rotating coordinates and metric from the PN metric near the PN failure point by adjusting the lapse $\alpha$ and shift $\beta_{j}$ so as to make the metric coefficients evolve on the inspiral timescale $\tau_{*}$. Subsequently $\alpha$ and $\beta_{j}$ must be chosen so as to make the coordinate time derivatives of all metric coefficients stay small, i.e., to make

$$
\mathcal{L}_{\partial_{t}} \boldsymbol{g} \simeq 0
$$

where $\mathcal{L}_{\partial_{t}} \boldsymbol{g}$ is the Lie derivative of the spacetime metric $\boldsymbol{g}$ with respect to the coordinate system's time generator $\partial_{t}$. To make this statement precise, we think of the left side of Eq. (2) as a velocity, construct a kinetic energy from this velocity, and choose a lapse and shift that minimize this kinetic energy. We will discuss several such action principles for $\alpha$ and $\beta_{j}$ in the next two subsections.

\section{A. The minimal-strain lapse and shift}

In the spirit of Smarr and York's [8] minimal distortion shift, we construct an action principle based on minimizing the Lie derivative of the spatial metric $\gamma_{i j}$ rather than the spacetime metric. Specifically, we presume that the numerical evolution has proceeded up to some slice of constant time $t$ that has intrinsic metric $\gamma_{i j}$ and extrinsic curvature $K_{i j}$, and we choose the lapse $\alpha$ and shift $\beta_{i}$ on this slice so as to minimize the positive definite action

$$
I_{1}\left[\alpha, \beta_{k}\right]=\int d^{3} x \sqrt{\gamma} \dot{\gamma}_{i j} \gamma^{i k} \gamma^{j l} \dot{\gamma}_{k l}
$$

Here $\dot{\gamma}_{i j}=\partial \gamma_{i j} / \partial t$ (the Lie derivative of $\gamma_{i j}$ along $\partial_{t}$ ) is expressed in terms of $K_{i j}, \alpha, \beta_{j}$ via

$$
\dot{\gamma}_{i j}=-2 \alpha K_{i j}+2 D_{(i} \beta_{j)},
$$

where $D_{i}$ is the spatial gradient compatible with the 3-metric $\gamma_{i j}$. By minimizing the action with respect to variations of $\alpha$ and $\beta_{i}$, we obtain four coupled equations:

$$
\begin{aligned}
& K^{i j}\left[-2 \alpha K_{i j}+2 D_{i} \beta_{j}\right]=0, \\
& D^{j}\left[-2 \alpha K_{i j}+2 D_{(i} \beta_{j)}\right]=0 .
\end{aligned}
$$

Equation (5a) is easily solved to give $\alpha$ in terms of $\beta_{j}$. When that $\alpha$ is inserted into Eq. (5b), the result is a linear, homogeneous differential equation for $\beta_{j}$. If the lapse were not fixed via Eq. (5a) but instead were chosen independent of $\beta_{j}$, e.g., via maximal slicing, then the shift equation (5b) would reduce to the minimal strain shift of Smarr and York [8]. We therefore refer to Eq. (5) as minimal strain equations.

Notice the geometrical nature of the spatial coordinates carried by this lapse and shift: The action principle (3) minimizes the rate of change, along $\partial_{t}$, of the infinitesimal proper distance between neighboring points at fixed spatial coordinates. This (presumably) will be achieved, in the binary itself, by making the coordinates co-rotate with the holes, and in the radiation zone by attaching the spatial coordinates to the wave pattern, i.e., by (almost) freezing the wave pattern into the spatial coordinate grid. A direct consequence is that evolution along $\partial_{t}$ is nearly shape and volume preserving.

In the IBBH problem, this approach is not without shortcomings: there is no guarantee that the minimal strain equations, which are solved on each spatial slice, will force the lapse and shift to evolve slowly. However, if the initial data are constructed in coordinates that are close to co-rotating (as they will be using the known PN metric), and if appropriate slow-change boundary conditions are enforced on $\beta_{i}$ near the holes' apparent horizons and at the outer edge of the coordinate grid, then it is reasonable to expect $\alpha$ and $\beta_{i}$ to evolve on the same slow timescale $\tau_{*}$ as the spatial metric $\gamma_{i j}$. This is because $\alpha$ and $\beta_{i}$ inherit their dynamics from the time evolution of $\gamma_{i j}$ and $K_{i j}$. Note that the minimal strain equations become degenerate for time-symmetric initial data; such a situation will not arise in the IBBH problem. A method of enforcing a variant of Eq. (5a) where $K^{i j}$ is replaced by $\gamma^{i j}$ has been explored by Balakrishna et al. [9].

The following (far from rigorous) argument makes it seem likely that this scheme will succeed for the IBBH problem. The IBBH spacetime has an "almost Killing vector field" $\boldsymbol{\xi}$, which embodies co-rotation and satisfies

$$
\mathcal{L}_{\xi} \boldsymbol{g} \equiv \boldsymbol{s} \sim \lambda / \tau_{*} .
$$

Here $\lambda \sim M$ is the length scale over which the spacetime curvature varies, and $\tau_{*} \gg \lambda$ is the inspiral timescale, so $s$ is small. In terms of the $3+1$ spacetime foliation being generated by the minimal-strain lapse and shift, we decompose $\boldsymbol{\xi}$ into a spatial piece $\boldsymbol{B}$ and a piece in the direction $\hat{\boldsymbol{n}}$ normal to the surfaces of constant $t: \boldsymbol{\xi}=A \hat{\boldsymbol{n}}+\boldsymbol{B}$, where $\boldsymbol{B} \cdot \hat{\boldsymbol{n}}=0$ by definition. We wish to determine the effectiveness of the minimal strain equations at attaching the coordinate grid to $\xi$, i.e., 
at making $\boldsymbol{\xi}=\partial_{t}$ or equivalently $A=\alpha$ and $\boldsymbol{B}=\boldsymbol{\beta}$. First, we project Eq. (6) into the spatial slice $\Sigma$ to get

$$
2 D_{(i} B_{j)}-2 A K_{i j}=s_{i j} .
$$

Next, because $\alpha$ and $\beta^{i}$ satisfy Eq. (5),

$$
D^{j}\left[-2 K_{i j} K^{k l} D_{k} \beta_{l} / K_{m n} K^{m n}+2 D_{(i} \beta_{j)}\right]=0 .
$$

Finally, substituting $\beta^{i}=B^{i}-b^{i}$ into this equation and using Eq. (7) we find that $b^{i}$, the difference between the minimalstrain shift and the shift we would like, satisfies

$$
\begin{aligned}
D^{j} & {\left[-2 K_{i j} K^{k l} D_{k} b_{l} / K_{m n} K^{m n}+2 D_{(i} b_{j}\right] } \\
& =D^{j}\left[-K_{i j}\left(K^{k l} s_{k l} / K_{m n} K^{m n}\right)+s_{i j}\right] .
\end{aligned}
$$

Assuming (without proof) that the boundary value problem for Eq. (8) is well posed, we see that there exists a solution to Eq. (9) that scales as $b^{i} \sim \lambda / \tau_{*}$; Eq. (5a) then implies that $\alpha-A \sim \lambda / \tau_{*}$. Therefore, the minimal-strain shift and lapse can make $\partial_{t}$ equal to the almost Killing vector field $\boldsymbol{\xi}$ that embodies co-rotation, aside from fractional differences of or$\operatorname{der} \lambda / \tau_{*}$, as desired.

Notice that if $\boldsymbol{\xi} \equiv A \hat{\boldsymbol{n}}+\boldsymbol{B}$ is a Killing vector field on the spacetime then $s_{i j} \equiv 0$, and $b^{i}=0$ is a trivial solution to Eq. (9) corresponding to $\alpha=A$ and $\beta^{i}=B^{i}$.

\section{B. Other choices of lapse and shift}

There is much freedom in choosing the lapse and shift to achieve the goal of slowly evolving metric coefficients. Another class of action principles that might work is based on minimizing an integral over spacetime rather than over 3space as in Eq. (3). Let $\partial_{t}=\alpha \hat{\boldsymbol{n}}+\boldsymbol{\beta}$ be the vector field to which our coordinates are tied, and denote the Lie derivative of the 4-metric along $\partial_{t}$ by $j_{\mu \nu}=\mathcal{L}_{\partial_{t}} g_{\mu \nu}$. Let $\boldsymbol{v}$ be some other vector field independent of $\partial_{t}$, and from it construct the tensor $H_{\boldsymbol{v}}^{\mu \nu}=g^{\mu \nu}+v^{\mu} v^{\nu}$. Then our class of actions is

$$
I_{2}\left[\partial_{t} ; \boldsymbol{v}\right]=\int_{\mathcal{M}}\left(j_{\mu \nu} H_{\boldsymbol{v}}^{\mu \rho} H_{\boldsymbol{v}}^{\nu \sigma} j_{\rho \sigma}\right) .
$$

On varying $\partial_{t}$, while holding $\boldsymbol{v}$ and the spacetime metric fixed, we arrive at

$$
\nabla_{\nu}\left(H_{v}^{\mu \rho} H_{v}^{\nu \sigma} j_{\rho \sigma}\right)=0
$$

This is a dynamical system of equations for the lapse and shift. Certain values of $\boldsymbol{v}$ might be considered most natural. If $\boldsymbol{v}=\sqrt{2} \times$ (some unit timelike vector), then $H_{\boldsymbol{v}}^{\mu \nu}$ is positive definite and there is a solution of Eq. (11) that truly minimizes the action. If $\boldsymbol{v}=0$, then Eq. (11) is a simple conservation law, but the action is not positive definite. It is trivial to show that a spacetime Killing vector field is a solution to Eq. (11) independent of the choice of $\boldsymbol{v}$, and straightforward to extend the analysis of Eqs. (7)-(9) to show that for the IBBH problem one of the solutions of Eq. (11) differs from the "almost Killing vector field"' of Eq. (6) by an amount that scales as $\lambda / \tau_{*}$. However, neither here nor for our minimal-strain equations have we managed to demonstrate that the desired solution for $\partial_{t}$ is an attractor; this needs further study.

\section{NUMERICAL EVOLUTION}

To fully solve the IBBH problem will require combining one of our methods to choose the lapse and shift with the Einstein equations in some concrete numerical scheme. Although the binary's metric coefficients should evolve on the long timescale $\tau_{*}$ in our proposed co-rotating coordinate system, there is danger that the time steps will be driven down to less than the size of the spatial grid by the numerical scheme's attempt to follow spurious gravitational waves and/or to control numerical instabilities (the Courant condition). To avoid these pitfalls while taking time steps controlled only by the inspiral timescale $\tau_{*}$, it will be necessary to stabilize the integration scheme and freeze out the degrees of freedom that are physically present but unphysically excited.

\section{A. Freezing out unwanted degrees of freedom}

It is well known that implicit differencing schemes freeze small-scale structures and produce unconditionally stable evolutions. For this reason, we envisage using an implicit scheme to evolve the Einstein equations in co-rotating coordinates.

While implicit differencing may be sufficient to achieve a stable evolution on the timescale $\tau_{*}$, we propose an additional technique that should also help. The idea is to convert the ADM equations for $\gamma_{i j}$ and $K_{i j}$ into a parabolic system, thereby removing all spurious waves while keeping the real ones (which are nearly frozen into the co-rotating coordinates).

The evolution equations in $\mathrm{ADM}$ form are

$$
\dot{\gamma}_{i j}=-2 \alpha K_{i j}+\mathcal{L}_{\beta} \gamma_{i j}
$$

$$
\dot{K}_{i j}=-D_{i} D_{j} \alpha+\alpha\left[{ }^{3} R_{i j}+\gamma^{k l}\left(K_{i j} K_{k l}-2 K_{i k} K_{l j}\right)\right]+\mathcal{L}_{\beta} K_{i j}
$$

where ${ }^{3} R_{i j}$ is the Ricci tensor constructed from $\gamma_{i j}$. This first-order system can be re-expressed as a second order system by solving Eq. (12a) for the extrinsic curvature and substituting it into Eq. (12b). Since the fields evolve on the very long timescale $\tau_{*}$ in the co-rotating frame, and since $\left(\partial_{t}\right)_{\text {inertial }} \simeq\left(\partial_{t}\right)_{\text {co-rotating }}+\Omega \partial_{\phi}$, the terms with two time derivatives in co-rotating coordinates will be smaller, by a factor $\sim 1 /\left(\Omega \tau_{*}\right)$, than at least some of those with a single time derivative. Thus, the double-time-derivative terms can be neglected (or back-differenced, if desired, so they are treated as sources arising from data on previous time slices). In particular, the term involving $\ddot{\gamma}_{i j}$ can be neglected (or backdifferenced). (Since $\alpha$ and $\beta_{i}$ are not dynamical fields, we suggest that their time derivatives also be back-differenced.) The resulting parabolic system of equations for $\gamma_{i j}$ can be evolved using an implicit scheme, which should be stable for large time steps. 


\section{B. Initial data and boundary conditions}

In addition to the evolution scheme, we must specify suitable initial data and boundary conditions. One can construct the initial data, just before the PN failure point, by using matched asymptotic expansions to join the post-Newtonian exterior metric onto the metrics of two tidally distorted Kerr black holes, and by then transforming to co-rotating coordinates [10].

The method of Cauchy characteristic matching (e.g., Ref. [11]) seems a promising candidate for constructing boundary data for the evolution of $\gamma_{i j}$ in the co-rotating frame. Such matching could conceivably be done around each of the holes and at an outer boundary in the radiation zone [12]. It may be possible to impose outgoing-wave boundary conditions as a constraint on the spatial derivatives of $\gamma_{i j}$ at the outer boundary. The shift there is $\boldsymbol{\beta} \simeq \Omega \partial_{\phi}$, where $\partial_{\phi}$ is the generator of rotations in the orbital plane, and outgoing waves are constant along $\partial_{r}+\boldsymbol{\beta}$ (aside from their $1 / r$ amplitude falloff), whereas spurious ingoing waves would be constant along $\partial_{r}-\boldsymbol{\beta}$.

We also need boundary conditions for the differential equation used to compute the shift. Fortunately, these seem easy to construct. Since the matching to characteristics would be done on the history of closed spatial 2-surfaces on the outer boundary and around each black hole, the derivations of the equations for the lapse and shift can be repeated for these surfaces. For example, the minimal strain equations, obtained by the variations of an action of the squared velocity of the metric on the 2-surface [the analogue of Eq. (3)], are

$$
\begin{gathered}
k^{a b}\left[-2 \alpha k_{a b}+2 \mathcal{D}_{a} \beta_{b}\right]=0, \\
\mathcal{D}^{a}\left[-2 \alpha k_{a b}+2 \mathcal{D}_{(a} \beta_{b)}\right]=0,
\end{gathered}
$$

where $k_{a b}$ is the extrinsic curvature of the 2-surface embedded in its history, $\mathcal{D}_{a}$ is the spatial gradient compatible with the metric on the 2-surface, and $\beta_{a}$ is the 3-dimensional shift vector projected into the 2 -surface. Since the boundary is closed, these equations can be solved to obtain the lapse function and the tangential components of the shift vector on the boundary. The normal component of the shift would be set to zero, so the evolution will not attempt to follow wave crests as they leave the numerical grid. Among the various solutions to Eqs. (13), the one we want will be that which is closest to the solution on the previous time slice. This boundary solution, combined with our 3-dimensional differential equations for the lapse and shift, presumably will produce the desired $\alpha$ and $\beta_{i}$, which evolve on the slow time scale $\tau_{*}$ and co-rotate with the holes.

\section{WORRIES}

Two conceivably serious difficulties with our approach are (i) in our co-rotating reference frame, the almost Killing vector becomes spacelike beyond the speed-of-light surface, which might cause problems for the evolution, and (ii) when the second time derivatives are discarded, the resulting evolution might not represent the true evolution of the space- time. We doubt, however, that these difficulties will actually arise, since we have seen no sign of them in a toy problem that retains the relevant features of the IBBH problem.

Our toy problem is a rotating, radiating sphere of scalar charge in flat spacetime. We adopt spherical polar coordinates $x^{i}=\{r, \theta, \phi\}$ and a line element given by Eq. (1) with $\gamma_{i j}=\operatorname{diag}\left[1, r^{2}, r^{2} \sin ^{2} \theta\right]$ and $\beta^{i}=0$. The sphere has radius $R$, angular velocity $\Omega(t)$, moment of inertia $I$ and scalar charge density $\rho[r, \theta, \phi-\Phi(t)]$ where $\Phi(t)=\int{ }^{t} d t^{\prime} \Omega\left(t^{\prime}\right)$. The scalar field $\Psi$ produced by this charge distribution satisfies the wave equation

$$
g^{\alpha \beta} \nabla_{\alpha} \nabla_{\beta} \Psi= \begin{cases}4 \pi \rho & \text { if } r<R \\ 0 & \text { otherwise. }\end{cases}
$$

As the sphere rotates, scalar waves are radiated to infinity, decreasing its angular momentum $I \Omega$ according to the radiation-reaction equation

$$
I \frac{\partial \Omega}{\partial t}=4 \pi \int_{\text {sphere }} d^{3} x \sqrt{\gamma}\left(\rho \partial_{\phi} \Psi\right) .
$$

For a quadrupolar distribution of scalar charge, separation of variables reduces the problem to solving a $(1+1)$ dimensional hyperbolic system; we evolved the system in the inertial frame using standard finite-difference methods and time steps constrained by the Courant condition.

We have also evolved this $1+1$ system in the co-rotating frame $\{r, \theta, \bar{\phi}=\phi-\Phi(t), t\}$ with $\Phi(t)$ inferred from the radiation-reaction equation (15) and not from some variant of our minimal-strain equations. In this co-rotating evolution, we discarded the (small) second time derivatives of $\Psi$ and applied an implicit differencing scheme to the resulting parabolic system. We succeeded in making the time steps as large as the rotational timescale of the charged sphere (much larger than the Courant condition would allow), and we believe that the time steps could be made as large as the radiation reaction timescale (which is much larger than the rotational timescale), since the limiting factor was the simple outer boundary condition that we used. There were no numerical instabilities. Moreover, no numerical problems were encountered at the speed-of-light surface $r=(\Omega \sin \theta)^{-1}$ [and we expected none, since the transformation to co-rotating coordinates does not change the fact that the wave equation (14) is manifestly hyperbolic]. There was good agreement between the results computed in the inertial and co-rotating frames. Further details will appear elsewhere [13]. Additional evidence that evolution in rotating coordinates need not cause problems comes from the work of Bishop et al. [14].

Based on these results, it seems likely that an implementation of the methods presented here will allow a numerical evolution of a binary system through the IBBH phase. Since a better understanding of this phase is important-and perhaps critical-for the LIGO and VIRGO detection of waves from binary black hole systems, and since such systems are 
highly promising candidate sources for LIGO and VIRGO, we hope to inspire researchers in numerical relativity to address the IBBH problem via our proposed techniques or others.

\section{ACKNOWLEDGMENTS}

This work was supported in part by NSF grants AST9417371, AST-9731698 and PHY-9424337 and NASA grants NAGW-4268/NAG5-4351 and NAG5-6840. P.R.B. is grateful to the Sherman Fairchild Foundation for financial support, and J.C. is grateful for partial support from the Natural Sciences and Engineering Research Council of Canada. The authors thank Sam Finn for helpful discussions, and for helpful critiques of an earlier version of this manuscript, they thank Miguel Alcubiere, Bernd Bruegmann, Joan Centrella, Carsten Gundlach, Richard Matzner, Ed Seidel, Stuart Shapiro, and James York.
[1] For $M_{1} \simeq M_{2}$, the volume of the Universe that LIGO and VIRGO can search scales approximately as $M_{1}^{2.5}$, so $M_{1}$ $\simeq M_{2} \simeq 15 M_{\odot}$ BBH's can be seen through a volume 400 times larger than binary neutron stars. This is likely to more than make up for the lower formation rate of BBH's, and it means that the first BBH's seen are likely to be the rarer ones with $M_{1} \sim 10-20 M_{\odot}$ rather than the presumably more common ones with $M_{1}<10 M_{\odot}$. See V. M. Lipunov, K. A. Postnov, and M. E. Prokhorov, New Astron. 2, 43 (1997); also É. É. Flanagan and S. A. Hughes, Phys. Rev. D 57, 4535 (1998).

[2] S. Sigurdsson and L. Hernquist, Nature (London) 364, 423 (1993) argue that one BBH forms in every core-collapse start cluster and that the resulting $\mathrm{BBH}$ merger rate may be as high as $\sim 1$ per year in a sphere of radius $200 \mathrm{Mpc}$ (the distance to which the first LIGO and VIRGO interferometers can see with signal to noise ratio 5.5 if $M_{1} \simeq M_{2} \simeq 15 M_{\odot}$ ), but the rate could be much lower. V. M. Lipunov, K. A. Postnov, and M. E. Prokhorov, New Astron. 2, 43 (1997) argue for a rate ranging up to this same value based on the evolution of populations of massive main-sequence binaries in galaxies like our own, but S. F. Portegies Zwart and L. R. Yungelson, Astron. Astrophys. 332, 173 (1998), in a similar type of analysis, obtain a vanishing rate of such BBH mergers. The enhanced LIGO interferometers are expected to see a volume 1000 times greater than the initial ones, and thus a 1000 times higher event rate; see K. S. Thorne, in Critical Problems in Physics, edited by Val L. Fitch, Daniel R. Marlow, and Margit A. E. Dementi (Princeton University Press, Princeton, New Jersey, 1997), p. 167.

[3] L. Blanchet, B. Iyer, C. Will, and A. Wiseman, Class. Quantum Grav. 13, 575 (1996); L. Blanchet, Phys. Rev. D 54, 1417 (1996).

[4] L. S. Finn and K. S. Thorne identified the IBBH Problem in spring 1996 and organized an August 1996 workshop at Caltech to brainstorm on its solution.
[5] We take a $2 \%$ error in energy loss rate to signal PN failure because the remaining number of radians of inspiral waveform is $\Phi \gtrsim 100$, and an error of more than 2 radians will seriously affect the LIGO and VIRGO data analysis. Our values of $v$ at PN failure are independent of the black-hole spins and are inferred from calculations in the limit $\eta \equiv$ (reduced mass)/(total mass) $\ll 1$ [Figure 1 of T. Tanaka, H. Tagoshi, and M. Sasaki, Prog. Theor. Phys. 96, 1087 (1996); Figure 1 of H. Tagoshi, M. Shibata, T. Tanaka, and M. Sasaki, Phys. Rev. D 54, 1439 (1996)], extrapolated to $\eta=1 / 4$ (equal masses). Our values of $T, N_{\text {orbits, }}$ and $\Phi$ are based on the Newtonian-quadrupole equations $T=(f M / 256 \eta) \nu^{-8}, N_{\text {orbits }}=(1 / 64 \pi \eta) \nu^{-5}$, and $\Phi$ $=4 \pi N_{\text {orbits }}$ with $\eta=1 / 4$, which are known to be accurate to within a few tens of percent at $\nu=0.3$ and $\eta \ll 1$ [L. S. Finn and K. S. Thorne (in preparation)].

[6] T. Damour, B. R. Iyer, and B. S. Sathyaprakash, Phys. Rev. D 57, 885 (1998).

[7] T. W. Baumgarte et al., Phys. Rev. Lett. 79, 1182 (1997); P. Marronetti, G. J. Mathews, and J. R. Wilson, gr-qc/9803093; S. Bonazzola, E. Gourgoulhon, and J. A. Marck, Phys. Rev. D 56, 7740 (1997).

[8] L. Smarr and J. W. York, Phys. Rev. D 17, 2529 (1978).

[9] J. Balakrishna et al., Class. Quantum Grav. 13, L135 (1996).

[10] K. Alvi (in progress); see also P. D. D'Eath, Phys. Rev. D 12, 2183 (1975).

[11] N. Bishop, R. Gomez, L. Lehner, B. Szilagyi, J. Winicour, and R. Isaacson, to appear in On the Black Hole Trail, edited by B. Iyer and B. Bhawal (Kluwer, Dordrecht, in press), gr-qc/9801070.

[12] R. Gomez, R. L. Marsa, and J. Winicour, Phys. Rev. D 56, 6310 (1997).

[13] P. R. Brady, J. Creighton, and A. G. Wiseman (in preparation).

[14] N. T. Bishop et al., Phys. Rev. D 56, 6298 (1997); R. Gomez, L. Lehner, R. L. Marsa, and J. Winicour, ibid. 57, 4778 (1998). 